\title{
Los entornos virtuales de aprendizaje como instrumento de mediación.
}

\author{
Cristóbal Suárez Guerrero \\ dvirtu22cusal.es \\ Universidad de Salamanca
}

"Toda actividad depende del material con el que opera"

Lev Vigotsky

\section{Introducción}

La educación es un proceso social por naturaleza, un evento que al estar implicado en una red de influencias mutuas, es indudablemente, el suceso más humano y humanizador de todas las finalidades sociales. Pero, ¿qué ocurre cuando esta constante y afortunada acción social discurre y se apoya sobre los hombros de las Nuevas Tecnologías de la Información y de la Comunicación (NTIC), que son hoy una variante? Evidentemente la representación de la acción educativa, así como las condiciones necesarias en el aprendizaje, se transforman. Este proceso se debe a que estas tecnologías están generando nuevas percepciones y oportunidades en los múltiples ámbitos de las relaciones sociales, y con ello, en la dinámica de la vida diaria de hoy, planteando por ello, un reto constante de redefinición a las iniciativas educativas en todos los niveles a escala mundial. Por tanto, la incorporación de las NTIC, como un nuevo componente del modelo pedagógico, exige a la teoría educativa umbrales particulares de análisis y compresión de la eficacia de estos nuevos instrumentos en el aprendizaje. Determinar este alcance educativo es imponderable en la actualidad, ya que no todo lo tecnológicamente viable es educativamente pertinente per se, necesitando añadir a este mundo de oportunidades, una dimensión pedagógica apropiada y necesaria a su vez.

Consecuentemente, desde esta necesidad por ensayar nuevos lenguajes o marcos de inteligibilidad pedagógicos sobre la presencia de las NTIC en la acción educativa, podemos plantearnos ahora una cuestión más específica: ¿qué es lo que cambia en la relación educativa cuando ésta se despliega bajo el auspicio de las NTIC? La certidumbre de la que partimos para responder la pregunta anterior se funda en que las NTIC, al mediar la relación educativa, transforma la relación. Esta modificación se genera a partir de una estructura básica de acción tecnológica que facilita el procesamiento, gestión y distribución de la información, agregando a la relación educativa, nuevas posibilidades y limitaciones para el aprendizaje. Esta transformación de la relación educativa promovida por la tecnología merece una atención distinta.

Por ello, a partir de la noción de mediación instrumental, se puede advertir que los instrumentos infovirtuales que participan en los procesos educativos, no pueden distinguirse sólo como simples artilugios tecnológicos u objetos impolutos culturalmente, sino que deben estimarse como auténticas estructuras de acción externa, pero además, como modelos para la reconfiguración de los marcos de pensamiento del sujeto. Esto es, un instrumento infovirtual regula y transforma tecnológicamente la relación educativa de un modo definido otorgando a los sujetos formas de actuación externa para el aprendizaje, pero a su vez, a partir de esa misma estructura y atributos tecnológicos, promueve en el sujeto una modificación interna de sus estrategias de pensamiento y aprendizaje. Esta doble orientación, externa e interna, atribuible a los instrumentos de mediación, debe representar otro punto de inflexión en el análisis y lectura pedagógica de las nuevas tecnologías en la relación educativa.

Consecuentemente, lo que pretendemos en las siguientes líneas es identificar cómo opera un instrumento infovirtual, como es el caso de los Entornos Virtuales de Aprendizaje (EVA), si se percibe 
como un instrumento de mediación educativo. Este análisis forma parte de una orientación pedagógica que busca explicar el alcance de las acciones educativas con estas tecnologías, así como reconocer cómo influyen en los componentes tácticos de la actividad mental en pro del aprendizaje. Además, esta insistencia pedagógica está alentada por la necesidad de completar el vacío teórico que exhiben las iniciativas de teleformación en la actualidad, que emplean muchos de estos entornos virtuales de aprendizaje los cuales, en el mejor de los casos, adolecen de un fundamento pedagógico sólido, y en el peor de las situaciones, simplemente están desprovistos de este fundamento (Sangrà, 2001; Echeverría, 2000). Por tanto, la tarea es ofrecer a partir de la evidencia de que estas tecnologías operan como instrumentos de mediación, una perspectiva pedagógica que ayude a orientar pertinentemente el aprendizaje, máxima finalidad de esta inserción tecnológica en el campo educativo, en estos contornos de virtualidad.

\section{Los instrumentos de mediación}

Para iniciar el recorrido es necesario destacar aquí algunos puntos imprescindibles desde el marco de percepción sociocultural del aprendizaje. Desde las formulaciones teóricas iniciadas por Vigostky, los procesos mentales superiores, es decir, los procesos estrictamente humanos se consideran de manera general, funciones de la actividad mediada. Esto es, el vector de análisis para entender el desarrollo hacia las funciones mentales superiores está dado a partir de la comprensión de que la internalización, la reconstrucción interna de una actividad externa, es posible gracias a la regulación que ejercen los instrumentos culturales de mediación en los sujetos en contextos sociales de relación. La mediación, por tanto, es el tema central en esta perspectiva psicológica que supone la actividad mediadora como principio constructor de lo específicamente humano: la cognición. Por ello, para el marco de la teoría sociocultural, la actividad humana no está entendida como simple respuesta o reflejo frente a un estimulante, la actividad implica un componente de transformación regulado a partir de los instrumentos -simbólicos y físicos- que la cultura proporciona al sujeto en interacción, y que tienen además, la particularidad de mediar la relación del sujeto con el mundo, con los hombres y consigo mismo.

La presencia de la actividad instrumental en la cognición es fundamental. No se trata pues de una comprensión accesoria de la participación de los instrumentos de mediación, sino que para la concepción sociocultural no existe desarrollo de los procesos mentales superiores sin la presencia de la actividad instrumental. Este eslabón no se encuentra en el individuo, sino fuera de él, en la cultura, que ha ido aquilatando durante generaciones formas colectivas históricamente determinadas y productos socialmente estructurados que toman forma de instrumentos, que nos construyen o nos conforman en condiciones de interacción con otros que nos la acercan. En ese sentido, nuestra mente no es una entelequia sumida en un vacío social ni un despliegue de un espíritu previo, es ante todo una entidad instrumentalizada (léase mediatizada) culturalmente y que se ajusta transformando activamente -de ahí las posibilidades de educabilidad- los insumos culturales dentro de una dinámica social. Como dice Bruner $(1999,12)$, "la cultura forma parte de la mente que nos aporta la caja de herramientas a través de la cual construimos no sólo nuestros mundos sino nuestras propias concepciones de nosotros mismos y nuestros poderes". Sin embargo, la instrumentalización de la mente no debe ser entendida sólo como una metáfora de comprensión, sino que debe ser concebida como la explicación de su desarrollo. Esto es, el proceso por el cual añadimos un componente de transformación o una nueva forma de organizar -regular- nuestra cognición, es posible en virtud de los instrumentos de mediación.

Pero, concretamente ¿cómo son y cómo actúan esos instrumentos? En la mediación instrumental se pueden identificar, a partir del criterio de actividad, por lo menos dos formas instrumentales de mediación: las herramientas y los signos; cada una orienta la actividad en un sentido. La diferencia esencial entre signo y herramienta, es decir, la razón para su entendimiento como dos líneas o modos de influencia de la actividad mediada que orientan la actividad humana, como describe Vigotsky $(2000,91)$, se esboza de la siguiente forma:

"La función de la herramienta no es otra que la de servir de conductor de la influencia humana en el objeto de 
la actividad; se halla externamente orientada y debe acarrear cambios en los objetos. Es un medio a través del cual la actividad humana externa aspira a dominar y triunfar sobre la naturaleza. Por otro lado, el signo no cambia absolutamente en nada en el objeto de una operación psicológica. Así pues, se trata de un medio de actividad interna que aspira a dominarse a sí mismo; el signo, por consiguiente, está internamente orientado".

Es decir, se puede ver al signo (como puede ser el lenguaje, los sistemas de numeración, los sistemas de lecto-escritura, sistemas convencionales legales, una estructura hipertextual, etcétera) y a la herramienta (dígase un hacha, un puente, un satélite, un ordenador, etcétera) como dos líneas de influencia precisas, pero a su vez, complementarias, ya que participan en un mismo proceso de conformación del sujeto.

En este sentido, la presencia de estos instrumentos en la actividad humana no es pasiva, por el contrario, es activa en estos dos sentidos. Por un lado, las herramientas están orientadas hacia el exterior, usándolas para operar o actuar sobre el mundo, mientras que por otro lado, los signos tienen una orientación hacia el interior, como medio que procura una regulación de los procesos psicológicos. Esta última es el aspecto más importante de la acción de los instrumentos sobre el sujeto. La doble orientación señalada, de lo físico y psicológico en un mismo instrumento de mediación, también puede entenderse bajo el término de artefactos, que son, como indica Cole (1999, 136) "simultáneamente ideales y materiales. Coordinan a los seres humanos con el mundo y entre sí de una manera que combina las propiedades de las herramientas y de los símbolos". En consecuencia, tanto símbolo como herramienta, son parte de una misma realidad instrumental. No obstante en este trabajo, seguiremos identificando, a partir de la orientación de la actividad, estos dos sentidos de actividad para poder focalizar y describir mejor la actividad mediacional de los EVA.

Como se puede entrever, a esta doble orientación no se escapan las NTIC. Éstas, al ser tecnologías propias de una época y de una circunstancia social concreta, facilitan por tanto, un modo de actuación definida y, a su vez, promueven una forma particular de regulación interna en el sujeto. Así, analizando un EVA con estas categorías podemos asociar esta doble orientación de la siguiente forma: Un EVA, como herramienta, nos permite encaminar y controlar una forma de actividad externa, acción que depende de la forma en que tecnológica y pedagógicamente está constituida para operar durante el proceso de aprendizaje. En cuanto signo, un EVA regula la propia actividad de quien usa la herramienta modificando sus marcos de pensamiento a partir de situaciones especificas derivadas de la propia estructura de acción tecnológica, desde donde inclusive, se puede seguir generando otras formas de pensar y actuar. Por tanto, un EVA a través de su entramado tecnológico nos brinda una forma específica de operar externamente durante el proceso de aprendizaje, así como la posibilidad de modificación interna a partir de esa misma forma de plantear el aprendizaje.

Esto nos sugiere asumir que un EVA no debe considerase como una entidad neutral o discreta, sino como una entidad que propone y permite una forma particular de aprender, y que a su vez, regula el pensamiento de quienes operan con, o a través, de ellos. Si esto es así, entonces un EVA, al ser el único ámbito educativo para el aprendizaje entre alumnos físicamente distantes, como normalmente ocurre en la teleformación, el entorno de interactividad debe ser considerado no sólo en su función de apoyo o material educativo -aunque siga siendo un material-, sino que debe repararse en él como una estructura que posibilita una acción externa y además como germen para la representación interna. Es necesario, por ende, reconocer que la función mediacional de un EVA en la teleformación es mayor, cuando se plantea como único medio o soporte para el aprendizaje.

Por lo tanto, desde la exigencia por delinear una dimensión pedagógica, un EVA debe ser considerado no sólo como un artilugio infovirtual, sino como un instrumento de mediación que propone una estructura de acción específica para aprender y, desde donde, cada alumno representa sus oportunidades y estrategias para el aprendizaje tecnológicamente mediado. En ese sentido, las tecnologías que participan en un proceso educativo pueden considerarse, como sistemas de actuación (acción externa), pero también, como fuente para la generación de nuevos modelos cognitivos o marcos de pensamiento (representación interna). Como advierte Vigotsky (2000, 92), "el uso de medios artificiales, la transición a la actividad mediata, cambia fundamentalmente todas las funciones 
psicológicas, al tiempo que el uso de herramientas ensancha de modo ilimitado la serie de actividades dentro de las que operan las nuevas funciones psicológicas". La actividad de aprendizaje no es ajena al material con el que se actúa, es más, nos conforma.

A continuación describiremos escuetamente este doble modo de actuación que puede generar un EVA para el desarrollo del aprendizaje, es decir, como un sistema de acción tecnológico definido, y luego, concluiremos con unas breves descripciones de los efectos que la tecnología podría generar en los marcos de pensamiento de los alumnos. Saber cómo se puede aprender, y en qué aspectos reconfigura la estrategia de pensamiento al aprender, debe ser un criterio necesario al momento de diseñar y proponer actividades educativas a través de estos instrumentos de mediación tecnológicos, como son los EVA, en la teleformación.

\section{Un EVA propone una estructura de acción tecnológica para el aprendizaje}

Para delimitar el primer aspecto con algún detalle, debemos destacar la ontología de la tecnología que M. Á. Quintanilla $(1989,34)$ precisa sobre la noción de realización técnica, definiéndola como "un sistema de acciones humanas intencionalmente orientado a la transformación de objetos concretos para conseguir de forma eficiente un resultado valioso". Como señalábamos, cuando optamos por una tecnología, no estamos optando simplemente por un aparato a secas, sino que optamos por todo lo que le incumbe como sistema de acción predeterminado, es decir, por un todo integral en el que se incluyen los componentes materiales, agentes intencionales, la estructura del sistema, los objetivos y los resultados[1] (Quintanilla, 1998). Extendiendo esto a un EVA, por definición, sería entonces: un sistema de acción que basa su particularidad en una intención educativa y en una forma específica para lograrlo a través de recursos infovirtuales. Esto es, un EVA orienta una forma de actuación educativa dentro de unos márgenes tecnológicos.

Pero, ¿cuál es el rasgo novedoso de actuación que imprime un EVA como sistema de acción tecnológico? Aquí indicaremos algunos de los aspectos relativos a esa nueva forma de orientar la acción que nos proporcionan las NTIC, y con ello un EVA, que no son otros que las posibilidades de acceso a la información y a la comunicación de un modo original. Para graficar esto señalaremos, a manera de ejemplo, las nuevas posibilidades de acción que nos podemos permitir gracias a la digitalización -dimensión técnica- y a la estructura hipertextual -dimensión expresiva- que intentaremos relacionar con el aprendizaje -dimensión pedagógica- en los EVA. Este aspecto, la imbricación dinámica de las dimensiones expresiva, técnica y pedagógica[2], constituyen aspectos necesarios en la caracterización estructural de un EVA (Suárez, 2002).

De esta forma, una de las posibilidades de acción que admite un EVA, el cual está basado en la capacidad tecnológica de la digitalización -aspecto que nos conduce al tema de la desmaterialización[3] del patrimonio cultural y socialmente estructurado-, permite que la realidad así digitalizada, pueda ser distribuida a cualquier parte del mundo (espacio) a velocidades instantáneas (tiempo). Es decir, existe una capacidad instalada de acción en las NTIC que permite que un emisor y un receptor -o viceversa- puedan compartir información, así como comunicarse, superando considerablemente las dificultades de ubicación y tiempo de manera eficiente.

Lo anterior es un hito en el replanteamiento de las relaciones humanas y, por lo tanto, en los modos de actuación educativa. Gracias a la capacidad infovirtual que despliegan los EVA, no sólo se modifican las formas de acceso a la información, sino que además se genera un contexto, un ámbito, particular de comunicación educativa. Este nuevo ámbito de operación o actividad basada en la tecnología proporciona al alumno una posición de control sobre las coordenadas espacio y tiempo, reformulando, a partir de esta condición, su perspectiva para la interacción. Esta reubicación conceptual en el aprendizaje generada por la digitalización, permite que a través de un EVA se minimice el concepto de distancia como impedimento para aprender, como también consiste que se pueda diversificar los tiempos para la interacción, pudiendo ser, como se sabe, síncrono o asíncrono.

Por otro lado, desde una dimensión expresiva, un EVA a través de su complexión hipertextual nos 
pone al alcance nuevos lenguajes para organizar el proceso de aprendizaje. Una estructura hipertextual, que es una constante en prácticamente todos los entornos de formación virtual a través de Internet, puede ser definida formalmente "como un grafo entre cuyos nodos existen relaciones de vinculación" (García-Valcárcel, 1996, 193). Gracias a esta estructura, la información adquiere una representación diferente a la forma secuencial o lineal de presentación a la que estábamos acostumbrados con otras tecnologías, como en el libro o el vídeo, por ejemplo. Esta representación nos otorga, por tanto, nuevos referentes de maniobra educativa, como a su vez, de reorganización de diversos tipos de información[4].

La reticularidad, por tanto, no sólo nos permite acceder a distintos tipos de información, sino que principalmente, nos permite organizarlas de manera similar a la inteligencia humana. Así, una estructura hipertextual, al facilitar el paso de un concepto o de un recurso a otro diferente a través de todo tipo de asociaciones libres "simula mejor el modo de proceder del entendimiento humano en el que tiene prioridad la reticularidad sobre la secuencia" (García Carrasco y García del Dujo, 2001, 396). Esta forma tecnológica de acción, de hecho, media el proceso de aprendizaje.

Se puede apreciar de esta manera que todo lo anterior no sólo implica una forma moderna o atractiva de operar a través de un EVA, son más bien, auténticas formas de acción que invitan a un proceder particular de exploración, organización y de gestión de la información, como de comunicación e interacción, que estimularían a su vez en los alumnos, la creación de un nuevo conjunto de estrategias conceptuales para el aprendizaje. Los EVA, por tanto, se manifiestan como una herramienta con una gran capacidad de modificación del entorno, y por tanto, de la acción educativa. No obstante, ¿cómo afectan estas estructuras de acción a las estructuras internas de los sujetos?

\section{A partir un EVA se puede representar marcos de pensamiento sobre el aprendizaje}

Se ha buscado constantemente poner en evidencia la forma cómo las tecnologías cambian nuestras mentes (Burke y Ornstein, 2001). Sin embargo, el marco de la teoría sociocultural, nos lleva a entender que la cognición, nuestra mente, no funciona sólo como una reacción biológica, sino que pasa a ser una entidad ampliada por el elemento cultural que modifica y conforma su estructura interna. Bajo esta orientación, autores como Pea (1999) prefieren hablar no sólo de la inteligencia como una propiedad de la mente, sino de la inteligencia como una propiedad ejecutada; es decir, mediatizada a través de los recursos externos, como son los instrumentos de mediación, que la cultura y la sociedad ponen a nuestro alcance.

Como venimos afirmando, las NTIC, hoy por hoy, insinúan una forma de mediación propia.

Consecuentemente, la tecnología infovirtual propone, sobre la base de su modo particular de sugerir la acción, modos diferentes de ejecutar la mente. Frente a esta idea global de que la mente está mediada, es decir, está amplificada por los instrumentos con los cuales el sujeto altera la realidad, debemos de advertir que cada instrumento cultural proporciona al sujeto una nueva, y no natural forma, de proyección o transfiguración de la realidad, y con ello, de sí mismo. Los EVA, que abrigan y soportan un sistema de acción infovirtual, son un tipo de instrumentos de mediación que afectan de una manera concreta los procesos internos de los sujetos que interactúan con o a través de ellos. Esta forma concreta de mediación está directamente asociada con la forma -compleja- en que procesan y distribuyen la información en el proceso de aprendizaje.

Este efecto mediacional de las nuevas tecnologías en el aprendizaje, según Salomon, Perkins y Globerson (1992), pueden ser de dos tipos: aquello que se puede aprender CON la tecnología, y aquello que se aprende DE la tecnología. Ambos son efectos mediacionales tecnológicos en los modos de aprender y pensar. Siguiendo esto, cuando se usan las tecnologías, como un EVA, para trabajar un tema de aprendizaje concreto es que podemos hablar de los efectos con la tecnología.

Recíprocamente, el otro sentido del efecto estaría asociado a las transformaciones cognitivas más o menos duraderas como consecuencia de la interacción con la estructura tecnología en sí, a este "residuo cognitivo" se le puede identificar como efectos de tecnología. La distinción es de suma 
importancia, ya que nos hace conscientes de estas dos formas de analizar las consecuencias en un proceso de interactividad persona-tecnología. No obstante, ambos efectos no deben ser percibidos como procesos inconexos, sino como parte de un mismo fenómeno que hay que identificar y valorar al momento de plantear los procesos de aprendizaje con tecnología.

Pero, ya que entendemos la idea de que la mente está mediada por los instrumentos a través de los cuales el sujeto reconstruye la realidad desde la posición añadida por la cultura, es cuando se hace inevitable preguntar, ¿cuáles son estos cambios en la mente atribuibles a la influencia mediadora del propio sistema de acción tecnológico, como puede ser un EVA? En otros términos, si estas tecnologías son producto de un conjunto de procesos cognitivos, entonces, ¿cuáles de esas funciones cognitivas resultan ser afectadas por sus propios productos?

Respecto a lo anterior, G. Salomon (1992) identifica una serie de sucedáneos cognitivos (efectos de tecnología) del uso de la tecnología informacional, que pone en evidencia que éstas, de hecho, modifican la forma de representación que tiene el pensamiento y que afectan específicamente lo que ha descrito como marcos de pensamiento[5] o componentes tácticos de la actividad mental. Estos vendrían a ser un conjunto de representaciones mentales -no estructuras constantes- que implican estrategias de pensamiento, estrategias de metacognición, formas de ver el mundo y ciertas habilidades de procesamiento y comunicación de la información, que efectivamente sirven de guía, apoyando y organizando, el proceso de aprendizaje. Esto es, los componentes tácticos para el aprendizaje se modifican a partir de situaciones específicas de interacción con la tecnología, es más, se modifican o ejecutan según la estructura de acción tecnológica que promueva. Salomon, en el mismo trabajo, señala que la influencia de la tecnología en la mente acontece por lo menos en cinco formas:

\footnotetext{
"a. La creación de metáforas que vienen a servir como "prismas cognitivos" a través de los cuales se examinan o interpretan los fenómenos; b. la estimulación de nuevas diferenciaciones con la consecuente creación de nuevas categorías cognitivas; c. la potenciación de la actividad intelectual; d. la potenciación de algunas de las habilidades específicas y la parcial extensión de otras; y, e. la internalización de modos y herramientas simbólicas tecnológicas que sirven como herramientas cognitivas."
}

Tomando en cuenta cada uno de los puntos citados anteriormente, se puede indicar como un EVA, al canalizar una acción cooperativa entre alumnos por ejemplo, influye en estos marcos de pensamiento. Inicialmente, al ser fuente de metáforas, la actividad a través de un EVA proporcionaría a los alumnos un re-encuadre del concepto de aula, llevándolo a nuevos linderos marcados por la constitución tecnológica de la que se vale un EVA para orientar la actividad educativa, como en el caso de la teleformación. Asimismo, un EVA puede auspiciar el desarrollo de nuevas diferencias conceptuales basadas en los modelos de actuación sugeridos como interacción recíproca entre alumnos; éstas son diametralmente diferentes a los propuestos en entornos diseñados sólo como depósitos de información. Por otro lado, los EVA pueden potenciar una forma renovada de comprender la interacción entre alumnos ya que la eleva exponencialmente a múltiples posibilidades -y limitacionesde comunicación que sólo pueden hacerse con esta tecnología y no con otras. También, con el uso de un EVA se pueden ver mejoradas -no descubiertas- una serie de habilidades cognitivas que dependen directamente del estímulo específico de cada herramienta, ampliando por tanto, el repertorio de lo que podemos pensar y hacer cooperativamente. Por último, se podrían internalizar representaciones simbólicas y herramientas complejas de actuación basadas en la interacción cooperativa entre personas, que podrían, en el mejor de los casos, extenderse más allá del perímetro de la teleformación hacia el desempeño profesional, por ejemplo.

No obstante, si estos marcos de pensamiento son un tipo de elementos cognitivos que implican y apoyan la generación de estrategias de pensamiento y de aprendizaje, se puede admitir la idea que las tecnologías computacionales, como los ordenadores, en el aprendizaje están mejor dispuestas si se usan como herramientas para la construcción del conocimiento, es decir, como herramientas de la mente (Jonassen, 2002). Sin desestimar la idea anterior, se debe considerar que en el caso de un EVA, al ser un entorno tecnológicamente enriquecido para aprender, contiene y sustenta elementos integrados que median la actividad como un sistema íntegro de actuación para el aprendizaje, es decir, 
como un sistema complejo de acción, no sólo como operaciones especificas. Por ello, la contundencia del efecto mediacional de un sistema de acción en la construcción del conocimiento, como en el aprendizaje, es mayor cuando: el sistema de actuación es más complejo y cuando se usa de manera exclusiva. Este es el caso de los EVA en la teleformación.

Si todo instrumento de mediación modifica siempre la orientación natural de la actividad, un EVA, reconociéndolo como herramienta de mediación supone también, un nuevo umbral para el desarrollo del aprendizaje. Por tanto, estos recursos externos, al orientar una acción, amplían nuestra concepción del qué, dónde y con quiénes se puede (y hasta es necesario) aprender. Es decir, los recursos infovirtuales en la relación educativa no sólo permiten que los alumnos puedan aprender, sino que al mismo tiempo, amplían las estrategias de aprendizaje. Como dice Claxton (2001), la tecnología implica un plus en el aprendizaje.

\section{Conclusión}

Los Entornos Virtuales de Aprendizaje (EVA) son en la actualidad el arquetipo tecnológico que da sustento funcional a las diversas iniciativas de teleformación. Sin embargo, desde su concepción, diseño y posterior empleo en los procesos de aprendizaje, los EVA deben satisfacer una visión pedagógica que enriquezca su constitución tecnológica inherente. Considerar este requerimiento puede orientar el uso de estas tecnologías más allá de los usos convencionales como simples máquinas, hacia una en que se contemple al aprendizaje como el principal motivo de su inclusión educativa.

Atendiendo esta demanda, desde la teoría sociocultural del aprendizaje, que logra poner de manifiesto que la mente no es una entelequia sumida en un vacío social, sino que lo propiamente humano se haya mediatizado, extendido si se quiere, a partir de la doble orientación de la actividad de los instrumentos de mediación, se puede destacar que las herramientas infovirtuales operan en el aprendizaje en dos sentidos. Los EVA al generar nuevos contextos o ámbitos de aprendizaje desde una estructura de acción tecnológica, posibilitan de manera recíproca, nuevos umbrales de representación cognitiva que influyen en las oportunidades de aprendizaje de quienes interactúan con estos instrumentos. Esta dinámica es de ida y vuelta, que hacen de los EVA un poderoso elemento de mediación educativa.

Por tanto, se debe comprender que todo aquello que se realiza a través de las herramientas infovirtuales, como sistema de actuación, interviene como condición de aprendizaje, y por ello, deja una secuela no sólo en aprendizaje de un tema, sino que influye en los marcos de pensamiento, esos componentes tácticos de actividad mental que orientan nuestras estrategias de aprendizaje. Por tanto, es necesario que la teleformación repare que un EVA añade un plus en el aprendizaje: no sólo se actúa con ella en el proceso de formación, sino que paralelamente se ejecuta la inteligencia, y con ella, las estrategias para aprender. No obstante, este influjo se hace más importante cuando, como en la teleformación, el medio es el que define el ámbito de actividad educativa.

Por ello, desde una perspectiva pedagógica hay que advertir que aprender dentro de los márgenes de virtualidad, debe suponer además, que esa virtualidad también nos conforma estructuralmente. Esta única visión respecto a una doble orientación puede, y debe, favorecer las propuestas educativas a través de los EVA, ya que se manifiestan como legítimas a su condición de instrumentos de mediación.

\section{Bibliografía}

BRUNER, J. (1999) La educación, puerta de la cultura. Madrid, Visor.

BURKE, J. y ORNSTEIN, R. (2001) Del hacha al chip. Cómo las tecnologías cambian nuestras mentes. Barcelona, Paidós.

COLE, M. (1999) Psicología cultural. Madrid, Morata. 
CLAXTON, G. (2001) Aprender. El reto del aprendizaje continuo. Barcelona, Paidós. En DARÍO, R. y otros (2000) Sobre herramientas cognitivas y aprendizaje colaborativo, V Congreso Iberoamericano de Informática Educativa.

[http://www.c5.cl/ieinvestiga/actas/ribie2000/papers/167]

DARÍO, R. y otros (2000) Sobre herramientas cognitivas y aprendizaje colaborativo, V Congreso Iberoamericano de Informática Educativa.[http://www.c5.cl/ieinvestiga/actas/ribie2000/papers/167/]

ECHEVERRÍA, J. (2000) Educación y nuevas tecnologías telemáticas, Revista Iberoamericana de Educación, 24.

[http://www.campus-oei.org/revista/rie24f.htm]

GARCÍA CARRASCO, J. y GARCÍA DEL DUJO, Á.( 2001) Teoría de la Educación II. Procesos primarios de formación del pensamiento y la acción. Salamanca, Ediciones Universidad de Salamanca.

GARCÍA-VALCARCEL, A. y TEJEDOR, F. J. (1996) (eds.) Perspectivas de las nuevas tecnologías en la educación. Madrid, Narcea.

JONASSEN, D. H., \& REEVES, T. C. (1996) Learning with Technology: Using Computers as Cognitive Tools, in JONASSEN, D. H. (Ed.) Handbook of Research on Educational Communications and Technology. New York, Simon \& Shuster Macmillan, 693-719.

JONASSEN, D. H. (2002) Computadores como herramientas de la mente. Eduteka. [http://www.eduteka.org/tema_mes.php3?TemaID=0012]

PEA, R. (2001) Prácticas de inteligencia distribuida y diseños para la educación, en SALOMON (comp.) Cogniciones distribuidas. Consideraciones psicológicas y educativas. Buenos Aires, Amorrortu.

QUINTANILLA, M. Á. (1989) Tecnología: un enfoque filosófico. Madrid, Fundesco.

QUINTANILLA, M. Á. (1998) Informe cultura tecnológica e innovación. Salamanca, Fundación COTEC.

SALOMON, G.; PERKINS, D. y GLOBERSON, T. (1992) Coparticipación en el conocimiento: al ampliación de la inteligencia humana con las tecnologías inteligentes, Comunicación, lenguaje y educación, 13, 6-22.

SALOMON, G. (1992) Las diversas influencias de la tecnología en el desarrollo de la mente, Infancia $y$ aprendizaje, 58, 143-159.

SANGRÀ, A. (2001) La calidad en las experiencias virtuales de educación superior, Actas de la conferencia internacional sobre educación, formación y nuevas tecnologías, 614-625.

SUÁREZ, C. (2002) Entornos virtuales de aprendizaje: interfaz de aprendizaje cooperativo. Trabajo de grado, Universidad de Salamanca.

VIGOTSKY, L. (2000) El desarrollo de los procesos psicológicos superiores. Barcelona, Crítica.

WALDEGG, G. (2002) El uso de las nuevas tecnologías para la enseñanza y el aprendizaje de las ciencias, Revista Electrónica de Investigación Educativa, 4 (1).

[http://redie.ens.uabc.mx/vol4no1/contenido-waldegg.html] 
[1] Estos cinco elementos del sistema de acción tecnológico pueden ser descritos así: componentes materiales (es la materia prima que se utiliza y se transforma en el sistema tecnológico), agentes intencionales (individuos), la estructura del sistema (las relaciones entre los componentes del sistema), los objetivos (las funciones para las que está hecho el sistema) y los resultados (el resultado de la acción intencional).

[2] Un aprovechamiento educativo de las ventajas de estas nuevas tecnologías nos debe orientar a consentir la existencia de una dimensión pedagógica. Esto es, la educación demanda un punto de referencia, un filtro de aprehensión, desde donde se pueda añadir a la operatividad instrumental que exhiben las NTIC, una representación educativa a favor del aprendizaje como su rasgo distintivo. Esta dimensión del uso educativo de las NTIC se debe articular dinámicamente con otras dos ya existentes en la naturaleza de estas herramientas: la dimensión técnica, en la que se puede agrupar propiedades como inmaterialidad, instantaneidad, calidad de imagen y sonido, digitalización, automatización, diversidad e interconexión (recursos y personas); y, la dimensión expresiva, en las que se puede identificar nuevos lenguajes como reticularidad, capacidad hipermedial y virtualidad. Todas estas dimensiones forman parte de un misma entidad.

[3] La desmaterialización es el proceso al que nos lleva la capacidad tecnológica de la digitalización. Gracias a este proceso, la realidad estructurada en átomos pasa a constituirse en esa realidad virtual constituida por bits ( 1 y 0 convertidos en impulsos) en el ordenador y que, a su vez, permite que esa realidad así estructurada convergentemente -otra cualidad técnica- de textos, gráficos, audio, video, etcétera, circule y pueda ser enviada a cualquier parte del mundo por las redes de información a la velocidad de la luz. Esta capacidad tecnológica posibilita no sólo nuevas formas de comunicación, sino que condiciona nuevas formas de relación social.

[4] Gracias a la WWW, que se basa en una estructura hipertextual (reticular), las posibilidades de maniobrar de manera indistinta en los innumerables ficheros de todos los servidores posibles a través de una serie de hiper-enlaces o lazos (links), convierten a Internet en una tupida red de información electrónica de nodos o nexos que dan acceso de manera integrada a textos, imágenes fijas, imágenes en movimiento, audio, gráficos, etcétera.... pero a su vez, ofrecen un conjunto de servicios de comunicación que, a partir de la misma interfaz, nos permiten transmitir información, como establecer relaciones sociales, a través de sus herramientas de comunicación como el e-mail, los foros, los chats, etcétera. Todo ello pone de manifiesto una forma específica de acción a través de los EVA.

[5] Hay que señalar que por marcos de pensamiento Salamon se refiere a un conjunto de representaciones mentales que orientan el pensamiento sirviendo de guía o catalizador en ese proceso. Un marco de pensamiento es una forma de organizar nuestra mente a través de diversas estrategias de pensamiento y aprendizaje. 